\title{
Utilidad de la escala TAPS para el rastreo del abuso de alcohol, tabaco y marihuana en atención primaria
}

\author{
Usefulness of the TAPS scale for the screening of abuse of alcohol, tobacco and marijuana in primary care
}

\section{Objetivos}

Valorar el desempeño de la herramienta Tabaco, Alcohol, Medicamentos recetados y otras Sustancias (TAPS según sus siglas en inglés) en pacientes de atención primaria.

\section{Diseño, lugar y pacientes}

Estudio multicéntrico que comparó la herramienta TAPS, autoadministrada y por entrevista, con una medida de referencia estándar, la Entrevista Diagnóstica Compuesta Internacional (en inglés Composite International Diagnostic Interview: CIDI) para evaluar el riesgo de uso problemático de sustancias (UPS) y de trastorno de consumo de sustancias (TCS).

Fueron incluidos 2000 mayores de 18 años que concurrieron a una consulta médica de cinco clínicas de atención primaria de adultos en EE.UU, entre agosto de 2014 y abril de 2015.

\section{Prueba de referencia}

Los autores usaron los ítems de la CIDI que se aplican a las clasificaciones de trastorno por consumo de sustancias del Manual

McNeely J y col. Ann Intern Med. 2016;165:690-699. Diagnóstico y Estadísticas de los Trastornos Mentales (en inglés: Diagnostic and Statistic Manual of Mental Disorders: DSM V). Se consideró UPS a un puntaje positivo en uno o más ítems de la CIDI y TCS, a un puntaje de dos o mayor.

\section{Prueba en estudio}

La TAPS se desarrolla en dos partes. La primera detecta el consumo de tabaco, alcohol, drogas ilícitas o medicaciones para uso no médico durante los últimos 12 meses. La segunda parte especifica la clase de sustancia consumida en los últimos tres meses.

Se consideró como punto de corte a una respuesta positiva para el UPS y dos para el TCS.

\section{Resultados}

Las características operativas de la TAPS se describen en la tabla 1.

Tabla 1. Sensibilidad y especificidad de la TAPS para la identificación del uso problemático y el trastorno por consumo de sustancias.

\begin{tabular}{|c|c|c|c|c|c|c|c|c|}
\hline & & Tabaco & Alcohol & Marihuana & $\begin{array}{c}\text { Cocaina y } \\
\text { metanfetamina }\end{array}$ & Heroina & Opiáceos & Sedativos \\
\hline $\begin{array}{l}\text { Uso problemático } \\
\text { de sustancias }\end{array}$ & $\begin{array}{c}\text { S } \\
\text { (IC 95\%) }\end{array}$ & $\begin{array}{c}0,93 \\
(0,9 a 0,95)\end{array}$ & $\begin{array}{c}0,74 \\
(0,70 \text { a } 0,78)\end{array}$ & $\begin{array}{c}0,82 \\
(0,76 \text { a } 0,87)\end{array}$ & $\begin{array}{c}0,68 \\
(0,59 a 0,77)\end{array}$ & $\begin{array}{c}0,78 \\
(0,67 \text { a } 0,87)\end{array}$ & $\begin{array}{c}0,71 \\
(0,58 \text { a } 0,82)\end{array}$ & $\begin{array}{c}0,63 \\
(0,47 a 0,78)\end{array}$ \\
\hline & $\begin{array}{c}\mathrm{E} \\
\text { (IC 95\%) }\end{array}$ & $\begin{array}{c}0,87 \\
(0,85 \text { a } 0,89)\end{array}$ & $\begin{array}{c}0,79 \\
(0,76 \text { a } 0,81)\end{array}$ & $\begin{array}{c}0,93 \\
(0,91 \text { a } 0,94)\end{array}$ & $\begin{array}{c}0,99 \\
(0,98 \text { a } 0,99)\end{array}$ & $\begin{array}{c}1 \\
(0,99 \text { a } 1)\end{array}$ & $\begin{array}{c}0,99 \\
(0,98 \text { a } 0,99)\end{array}$ & $\begin{array}{c}0,99 \\
(0,98 \text { a } 0,99)\end{array}$ \\
\hline $\begin{array}{l}\text { Trastorno por } \\
\text { consumo de }\end{array}$ & $\begin{array}{c}\text { S } \\
\text { (IC 95\%) }\end{array}$ & $\begin{array}{c}0,74 \\
(0,69 \text { a } 0,77)\end{array}$ & $\begin{array}{c}0,70 \\
(0,64 \mathrm{a} 0,75)\end{array}$ & $\begin{array}{c}0,71 \\
(0,63 \text { a } 0,79)\end{array}$ & $\begin{array}{c}0,57 \\
(0,47 \text { a } 0,67)\end{array}$ & $\begin{array}{c}0,66 \\
(0,53 \text { a } 0,77)\end{array}$ & $\begin{array}{c}0,48 \\
(0,33 \text { a } 0,63)\end{array}$ & $\begin{array}{c}0,54 \\
(0,34 \mathrm{a} 0,72)\end{array}$ \\
\hline sustancias & $\begin{array}{c}\mathrm{E} \\
\text { (IC 95\%) }\end{array}$ & $\begin{array}{c}0,89 \\
(0,88 \text { a } 0,91)\end{array}$ & $\begin{array}{c}0,85 \\
(0,83 \text { a } 0,87)\end{array}$ & $\begin{array}{c}0,95 \\
(0,94 \mathrm{a} 0,96)\end{array}$ & $\begin{array}{c}0,99 \\
(0,99 \text { a 1) }\end{array}$ & $\begin{array}{c}1 \\
\left(\begin{array}{l}1 \\
\mathrm{a}\end{array}\right)\end{array}$ & $\begin{array}{c}1 \\
(0,99 \text { a 1) }\end{array}$ & $\begin{array}{c}0,99 \\
(0,98 \text { a } 0,99)\end{array}$ \\
\hline
\end{tabular}

$\mathrm{S}=$ Sensibilidad $\mathrm{E}=$ Especificidad

\section{Comentario}

La detección del consumo de sustancias y la valoración del riesgo de dicho consumo es una labor fundamental para los equipos de atención primaria de la salud. Es frecuente que los pacientes depositen la confianza en dichos profesionales, ya sea para comentarles sus propios problemas relacionados al consumo o bien para hablar sobre los problemas de algún miembro de la familia. Por otro lado, es sabido que hay un elevado monto de negación en los pacientes consumidores y de normalización del consumo de algunas sustancias. Su detección es fundamental, así como también el registro del consumo en la historia clínica, ya que es el primer paso para interceder a través de intervenciones breves o, en los casos de consumo problemático, mediante la derivación para tratamiento en centros especializados ${ }^{1}$. Los instrumentos de cribado, tienen por objetivo la detección de "probables casos" de abuso o dependencia de sustancias y pueden ayudar a los profesionales de atención primaria cuando su administración es sencilla y no demanda mucho tiempo. Algunos de los más usados en nuestro medio son el AUDIT y el CAGE para la detección de consumo problemático de alcohol y el Fag- erström, para adicción al tabaco². Comparado con otros instrumentos de cribado usados para el consumo de sustancias, la TAPS valora el uso de varias sustancias, lo que posibilita su aplicación en contextos de atención clínica, lo que es facilitado por la opción de la versión autoadministrada para el paciente. Una desventaja que limita su aplicación en nuestro ámbito es que por el momento, sólo contamos con la versión en idioma inglés ${ }^{3}$.

\section{Conclusiones de la comentadora}

Es fundamental que el equipo de salud se informe y esté formado en la detección y el abordaje de los pacientes con adicciones, dado que constituyen un problema de salud cuya incidencia va en aumento. Las herramientas de rastreo pueden ser muy útiles para detectar a los pacientes consumidores y para valorar su riesgo.

Si bien la TAPS, al detectar principalmente las sustancias más consumidas en nuestra población, puede ser una herramienta efectiva, aún no ha sido utilizada en nuestro medio.

Cecilia De Simone [ Servicio de Psiquiatría y Programa de Control de Tabaco del Hospital Italiano de Buenos Aires. cecilia.desimone@ hospitalitaliano. org.ar ]

De Simone C. Utilidad de la escala TAPS para el rastreo del abuso de alcohol, tabaco y marihuana en atención primaria. Evid Act Pract Ambul. 2017;20(4). 99. Comentado de: McNeely J y col. Performance of the Tobacco, Alcohol, Prescription Medication and Other Substance Use (TAPS) Tool for Substance Use Screening in Primary Care Patients. Ann Intern Med. 2016;165:190-199. PMID: 27595276. 